Proceedings of the International School and Conference on Optics and Optical Materials, ISCOM07, Belgrade, Serbia, September 3-7, 2007

\title{
Effect of Laser Light Ellipticity on Hanle Electromagnetically Induced Absorption Amplitude and Line Width
}

\author{
J. Dimitrijević* Z Z. Grujić, M. Mijailović, D. Arsenović, \\ B. Panić And B.M. Jelenković
}

Institute of Physics, Pregrevica 118, 11080 Belgrade, Serbia

Using the ${ }^{2} S_{1 / 2} F_{\mathrm{g}}=2 \longrightarrow{ }^{2} P_{3 / 2} F_{\mathrm{e}}=3$ transition in ${ }^{87} \mathrm{Rb}$, we analyzed the interaction between the arbitrarily polarized laser light and Zeeman sublevels in the Hanle configuration. We studied theoretically and confirmed experimentally that the effect of the laser light polarization on the electromagnetically induced absorption strongly depends on the laser light power. For the same atomic transition, and for single laser light power $(3 \mathrm{~mW})$, it was recently shown that electromagnetically induced absorption line widths increase with polarization ellipticity and that electromagnetically induced absorption amplitude has maximum for certain laser light polarization different than linear. Here, we present results for amplitudes and widths of electromagnetically induced absorption for different laser light polarizations and for the laser light power $\leq 200 \mu \mathrm{W}$. It is verified that for small laser light power maximal electromagnetically induced absorption amplitude is for the linear polarization, whereas for larger power maximum of the electromagnetically induced absorption is at higher polarization ellipticity.

PACS numbers: 42.50.Gy, 32.70.Jz

\section{Introduction}

Coherence population trapping (CPT) [1], a phenomenon based on atomic coherence, has been intensely investigated over the past decade due to numerous applications of its manifestation. Electromagnetically induced transparency (EIT) [1] is manifestation of CPT, able to suppress light absorption of resonant multilevel atomic systems. Electromagnetically induced absorption (EIA) [2] is opposite phenomenon of EIT in a sense that absorption is enhanced. Physical origin of EIA is in the spontaneous transfer of the light induced anisotropy (atomic coherence or/and population difference) to the ground levels [3]. Typically, EIA is observed

*corresponding author; e-mail: jelenad@phy.bg.ac.yu 
in atomic systems where degeneracy of excited state is higher than of ground state, forming so-called V-schemes.

Although interaction with elliptically polarized light is important, there are very few results of EIA obtained with a laser light with various light ellipticity. Calculations of EIA profiles, for degenerate two-level system and for various combinations of linearly and circularly polarized probe and pump lasers, for different probe detuning were presented in [4]. Effect of ellipticity of the laser light on Hanle EIA was presented in [5] by an analytical solution of the model for $F_{\mathrm{g}}=1 \longrightarrow F_{\mathrm{e}}=2$ atomic transition. They show Doppler narrowing of the line widths for the same ellipticity, and narrowing with increased light ellipticity for the same Doppler width. More recently, the same group has shown strong dependence of EIA amplitude and line width on the light polarization in ${ }^{87} \mathrm{Rb}$, at high laser power $(P=3 \mathrm{~mW})[6]$.

In this study we extended the work of [6]. We made theoretical and experimental investigations of the EIA dependence on the laser ellipticity for laser power between 50 and $200 \mu \mathrm{W}$. Experiment was done in the Hanle configuration, by measuring laser transmission as a function of the external magnetic field $\left(B_{\text {scan }}\right)$ parallel to the laser propagation. Theoretically, calculations were done by solving optical Bloch equations and by averaging over Maxwell-Boltzmann velocity distribution.

\section{Theoretical model}

We solve optical Bloch equation to describe absorption of laser light locked to $F_{\mathrm{g}}=2 \longrightarrow F_{\mathrm{e}}=3$ transition in ${ }^{87} \mathrm{Rb}$. Properties of light enter the equations through electric field vector and it runs along $z$-axis. For a laser light of arbitrary ellipticity $\varepsilon$ (defined by $\tan \varepsilon=E_{(1) 0 y} / E_{(1) 0 x}$ ) it holds

$$
\boldsymbol{E}\left(\boldsymbol{r}_{0}, t\right)=\boldsymbol{e}_{x} \cos \left(\omega_{(1)} t\right) E_{(1) 0 x}+\boldsymbol{e}_{y} \cos \left(\omega_{(1)} t+\varphi_{(1)}^{y x}\right) E_{(1) 0 y} .
$$

We start from the equation

$$
\dot{\hat{\varrho}}=\frac{\mathrm{i}}{\hbar}[\hat{\varrho}, \hat{H}]-\sum_{l} \Gamma_{l}\left(\hat{A}_{l}^{\dagger} \hat{A}_{l} \hat{\varrho}-2 \hat{A}_{l} \hat{\varrho} \hat{A}_{l}^{\dagger}+\hat{\varrho} \hat{A}_{l}^{\dagger} \hat{A}_{l}\right)-\gamma\left(\hat{\varrho}-\hat{\varrho}_{0}\right),
$$

where $\hat{H}=\sum_{i} \hbar \omega_{\mathrm{g}_{i}}\left|g_{i}\right\rangle\left\langle g_{i}\left|+\sum_{i} \hbar \omega_{\mathrm{e}_{i}}\right| e_{i}\right\rangle\left\langle e_{i}\right|-e \boldsymbol{E}\left(\boldsymbol{r}_{0}, t\right) \hat{\boldsymbol{r}}$, with five ground $\left|g_{i}\right\rangle$ and seven excited $\left|e_{i}\right\rangle$ Zeeman sublevels. Applying rotating wave approximation leads to optical Bloch equations for our system. For matrix dipole moment, it holds

$$
\mu_{m_{\mathrm{e}}, m_{\mathrm{g}}, q}=e\left\langle m_{\mathrm{e}}\left|\boldsymbol{u}_{q} \boldsymbol{r}\right| m_{\mathrm{g}}\right\rangle=\mathcal{G}_{1}(-1)^{m_{\mathrm{e}}}\left(\begin{array}{ccc}
2 & 1 & 3 \\
m_{\mathrm{g}} & q & -m_{\mathrm{e}}
\end{array}\right),
$$

where $\boldsymbol{u}_{-1}=\frac{\boldsymbol{e}_{x}-\mathrm{i} \boldsymbol{e}_{y}}{\sqrt{2}}, \boldsymbol{u}_{+1}=-\frac{\boldsymbol{e}_{x}+\mathrm{i} \boldsymbol{e}_{y}}{\sqrt{2}}$ and $\boldsymbol{u}_{0}=\boldsymbol{e}_{z}$. $\mathcal{G}_{1}$ is the constant proportional to the reduced matrix element of the dipole operator between the ground and the excited states

$$
\mathcal{G}_{1} \sim\left\langle n_{\mathrm{e}} L_{\mathrm{e}}\|\boldsymbol{r}\| n_{\mathrm{g}} L_{\mathrm{g}}\right\rangle .
$$

Second term from the right hand side (rhs) of Eq. (2) describes spontaneous emission. The sum is over all allowed transitions and $\hat{A}_{e_{i} g_{j}}=\mu_{e_{i}, g_{j}, i-j}\left|g_{j}\right\rangle\left\langle e_{i}\right|$. The 
quantity $\Gamma=\frac{2}{7} \Gamma_{L}\left|\mathcal{G}_{1}\right|^{2}=2 \pi \times 6.07 \mathrm{MHz}$ is total spontaneous emission rate of any excited sublevel. Energy splitting due to applied magnetic field $B_{\text {scan }}$ is given by

$$
E_{\mathrm{g}(\mathrm{e})}=\mu_{\mathrm{B}} g_{F_{\mathrm{g}(\mathrm{e})}} m_{\mathrm{g}(\mathrm{e})} B_{\mathrm{scan}} .
$$

Here $\mu_{\mathrm{B}}$ is the Bohr magneton and $g_{F_{\mathrm{g}(\mathrm{e})}}$ is the Lande gyromagnetic factor for two hyperfine levels. The last term in rhs of the Bloch equation describes the rate at which atoms enter and leave the laser beam. We take $\gamma=\Gamma / 100$ and $\hat{\varrho}_{0}=\frac{1}{5} \sum_{i}\left|g_{i}\right\rangle\left\langle g_{i}\right|$. The atoms fly in the laser beam long enough so they reach steady state. Therefore we set time derivations in left hand side (lhs) of the Bloch equations to zero and then solve them as 144 linear algebraic equations. As a transmission spectroscopic signal we consider total ground state population

$$
\Pi_{g}=\sum \varrho_{g_{i} g_{i}} .
$$

The Doppler effect was taken into account assuming Maxwell-Boltzmann velocity distribution and by averaging contributions of atoms whose projections of velocities along laser light are in the interval $(-700,700) \mathrm{m} / \mathrm{s}$.

\section{Experimental setup}

The experimental setup used for measurements of EIA in the Hanle configuration is explained in detail in [7]. External cavity diode laser is frequency stabilized at $F_{\mathrm{g}}=2 \longrightarrow F_{\mathrm{e}}=3$ transition of ${ }^{87} \mathrm{Rb} D_{2}$ line. Laser beam radius is $4.07 \mathrm{~mm}$. The $1 \mathrm{~cm}$ long $\mathrm{Rb}$ vapor cell with pure ${ }^{87} \mathrm{Rb}$ and no buffer gas is inside a solenoid which produces variable magnetic field (longitudinal) in the same direction as laser beam, and all together is placed between 3 pairs of Helmholmtz's coils, in order to eliminate stray magnetic fields. Linear neutral density filter is used to achieve desired laser intensity. Laser field ellipticity is controlled by $\lambda / 4$ plate. The intensity of transmitted laser light is detected with photodiode and recorded by the digital oscilloscope together with voltage used to drive current supply for the solenoid.

\section{Results}

In the following figures we present theoretical and experimental results of EIA amplitudes and widths, derived from the Hanle profiles, when the laser's ellipticity changes from linear $\left(\varepsilon=0^{\circ}\right)$ to $\varepsilon=30^{\circ}$ and power varies in the range from $50 \mu \mathrm{W}$ to $200 \mu \mathrm{W}$. Results of the EIA amplitudes are shown in Fig. 1. Figures $1 \mathrm{a}-\mathrm{c}$ present experimental, while figures (d) to (f) present theoretical results of the EIA amplitudes as a function of the laser ellipticity, for three different laser light intensities.

Both calculated and measured results show that for small laser power $(P=$ $50 \mu \mathrm{W})$ maximum of the EIA amplitude is for linear polarization. Increase in laser power to $P=200 \mu \mathrm{W}$ shifts this maximum to ellipticity of $\varepsilon=10^{\circ}$. Results presented in [6] show maximal EIA amplitude at $\varepsilon=20^{\circ}$ for the laser light power $P=3 \mathrm{~mW}$. Therefore, for specific laser power, one needs laser light of particular 

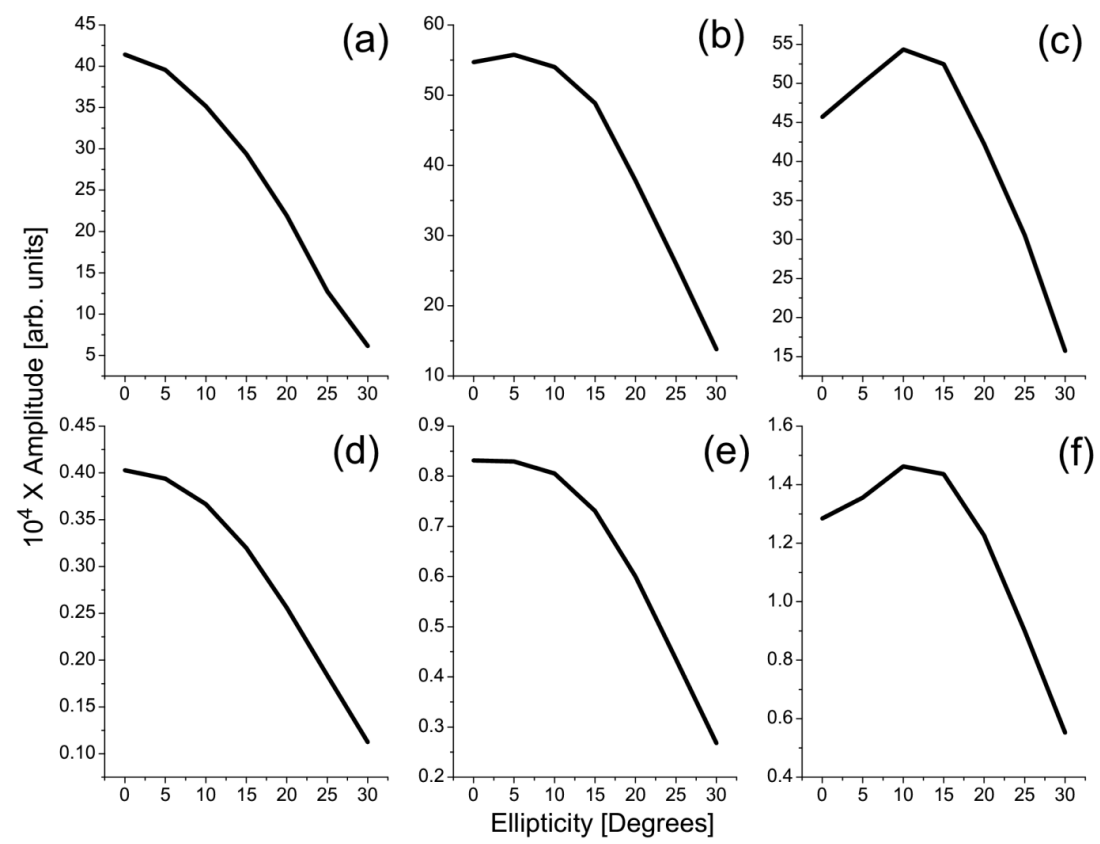

Fig. 1. Measured (top row) and calculated (bottom row) amplitudes; (a) and (d) $P=$ $50 \mu \mathrm{W}$; (b) and (e) $P=100 \mu \mathrm{W}$, and (c) and (f) $P=200 \mu \mathrm{W}$.

ellipticity in order to most efficiently generate the EIA. Such behavior of the EIA is different from EIT dependence as a function of the laser light ellipticity. Maximum of the EIT is always for linearly polarized light [5] regardless of the laser power.

Variation of the EIA widths as a function of the laser ellipticity, and for the same range of the laser power, are given in Fig. 2. Both theory and experiment predict similar values for EIA widths, between 180-300 mG. Let us note that our theoretical model gives widths in exact units of $B_{\text {scan }}$. Discrepancies between calculated and measured widths results may be due to the fact that theory did not take into account spatial variation of the laser power in the cell and the Gaussian profile of the laser beam.

It is apparent that efficiency of the laser light to induce EIA depends on the laser ellipticity, and that this dependence is different at different laser intensities. Higher efficiency of the light of elliptical polarization, vs. linearly polarized laser light, at higher laser intensities, can be explained by more efficient pumping to edged magnetic sublevels of the $F_{\mathrm{g}}=2$. Calculations of the population of the Zeeman sublevels as a function of the laser intensity for linearly polarized light [8] and for elliptically polarized light (not shown) tell that the observed signal depends on the level of optical pumping of the Zeeman sublevels of the ground hyperfine state (orientation). We would like to point out that calculations also show that the observed behavior of the EIA is under strong influence of the Doppler broadening. 


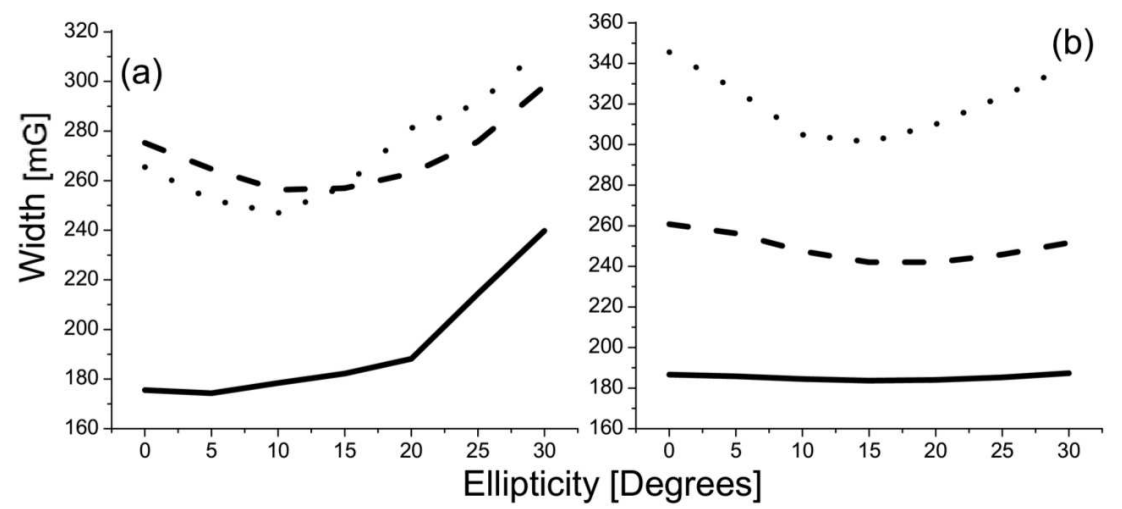

Fig. 2. Measured (a) and calculated (b) widths; solid lines $P=50 \mu \mathrm{W}$; dashed lines $P=100 \mu \mathrm{W}$; and dotted lines $P=200 \mu \mathrm{W}$.

In conclusion, we have demonstrated theoretically and experimentally influence of the laser intensity on efficiency of generation of "bright" states using laser light of various ellipticity. It was shown that for the low light intensity linearly polarized light is more efficient, while for higher laser intensity elliptically polarized light enhances EIA amplitudes in respect of linearly polarized light. Such behavior of the EIA as a function of the laser ellipticity and intensity is due to intensity dependent population of magnetic sublevels of the ground state hyperfine level.

\section{Acknowledgments}

This work was supported by the Ministry of Science and Environmental Protection of the Republic of Serbia, under grant number 141003.

\section{References}

[1] E. Arimondo, Prog. Opt. 35, 257 (1996).

[2] A.M. Akulshin, S. Barreiro, A. Lezama, Phys. Rev. A 57, 2996 (1998).

[3] A.V. Taichenachev, A.M. Tumaikin, V.I. Yudin, Phys. Rev. A 61, 011802(R) (1999).

[4] A. Lipsich, S. Barreiro, A.M. Akulshin, A. Lezama, Phys. Rev. A 61, 053803 (2000).

[5] D.V. Brazhnikov, A.M. Tumaikin, V.I. Yudin, A.V. Taichenachev, J. Opt. Soc. Am. B 22, 57 (2005).

[6] D.V. Brazhnikov, A.V. Taichenachev, A.M. Tumaikin, V.I. Yudin, S.A. Zibrov, Ya.O. Dudin, V.V. Vasilev, V.L. Velichansky, JETP Lett. 83, 64 (2006).

[7] M.M. Mijailović, J. Dimitrijević, A.J. Krmpot, Z.D. Grujić, B.M. Panić, D. Arsenović, D.V. Pantelić, B.M. Jelenković, Opt. Express 15, 1328 (2007).

[8] J. Dimitrijević, D. Arsenović, B.M. Jelenković, Phys. Rev. A 76, 013836 (2007). 